\title{
Aggregated states of luminescent conjugated polymers in solutions
}

\author{
R. Chang ${ }^{a}$, J.H. Hsu ${ }^{\mathrm{a}, \mathrm{b}}$, W.S. Fann ${ }^{\mathrm{a}, \mathrm{b}}$, J. Yu ${ }^{\mathrm{c}}$, S.H. Lin ${ }^{\mathrm{a}}$, Y.Z. Lee ${ }^{\mathrm{d}}$, S.A. Chen ${ }^{\mathrm{d}}$ \\ a Institute of Atomic and Molecular Sciences, Academia Sinica, P.O. Box 23-166, Taipei, 106 Taiwan \\ ${ }^{\mathrm{b}}$ Department of Physics, National Taiwan University, Taipei, Taiwan \\ ${ }^{\mathrm{c}}$ Department of Chemistry, National Kaoshiung Normal University, Kaoshiung, Taiwan \\ ${ }^{\mathrm{d}}$ Department of Chemical Engineering, National Tsing-Hwa University, Hsinchu, Taiwan
}

Received 18 March 1999; in final form 14 September 1999

\begin{abstract}
In order to study the electronic properties of the interchain state in light-emitting polymers, we have performed spectroscopic experiments for 2,5-dioctyloxy PPV (DOO-PPV) in both good (chloroform) and poor (2-methyl-tetrahydrofuran) solvents. The concentration-dependent absorption, photoluminescence (PL) spectra and dynamics of time-resolved PL can be explained by the coexistence of an intrachain and one kind of aggregated states in poor solution. The estimated lifetime and transition moment of the aggregated state lead to significant quantum efficiency of PL which is about 0.75 times that of the intrachain state. The PL spectrum for the aggregated state resembles that of a thin film, implying that the emissive species in thin film is more likely to be an aggregated state. (c) 2000 Elsevier Science B.V. All rights reserved.
\end{abstract}

\section{Introduction}

Light-emitting polymers such as PPV and its derivative have been extensively applied in recent years [1-4]. However, numerous fundamental issues must be clarified, the most important one being the existence of an interchain effect. Rothberg et al. [5-7] proposed that the main part of the excitation in PPV film is the interchain non-emissive excitons, i.e. indirect excitons or bound poloran pairs. Since then, this model has been widely discussed [8-12].

In addition, aggregated states have received increasing attention [13-18]. Bässler et al. investigated the LPPP, a $\pi$-conjugated system, indicating that the photoluminescence (PL) of LPPP thin film acquires an additional low-energy band than that of the solution phase. By using site-selective fluorescence, they ascribed the above observation to the existence of aggregation in the LPPP film instead of the excimer. Excimers refer to the dimers which are formed only in the excited states, while dissociated in the ground state, and also have been observed in some lightemitting polymers [18]. In CN-PPV, Samuel et al. [14-16] observed that PL in thin films becomes red-shifted, broad and structureless compared with solution phase. They also concluded the existence of aggregated states in the CN-PPV thin film as well as poor solution.

In light of the above developments, Hsu et al. [19] spectroscopically examined 2,5-dioctyloxy PPV (DOO-PPV) in poor solution and solid phase and deduced the existence of an aggregate state. In this Letter, we study DOO-PPV in poor solution while varying the concentration. In contrast to thin film, 
poor solution is an environment that could lead to the formation of interchain states with much less variety, hence the analysis becomes relatively simple. The most striking result is that the absorption and fluorescence spectra in the poor solvent can be completely described by two emissive species. The spectral characters of these two species are independent of concentration variation of two order of magnitude. We therefore infer that the intrachain and aggregated state coexist in solution of high concentration. The latter has an energy lower than the former by $1300 \mathrm{~cm}^{-1}$ and decays with a shorter lifetime. This finding is different from the assumption that interchain interactions only increase the conjugation length of the intrachain exciton [20] since the latter would cause a substantially small energy shift. Meanwhile, we also find that, with respect to the intrachain state, the quantum efficiency of the aggregated state is about 0.75 .

\section{Experimental}

DOO-PPV, with its structure schematically depicted in Fig. 1, was prepared following a procedure similar to that used by Holmes et al. [21] and Wudl et al. [22]. The molecular weight of DOO-PPV in THF solution was determined to be about 10000 by using gel permeation chromatography (GPC). The poly-dispersity is 2.23 . The solubility of DOO-PPV in 2-methyltetrahydrofuran (m-THF) is 'poor' at

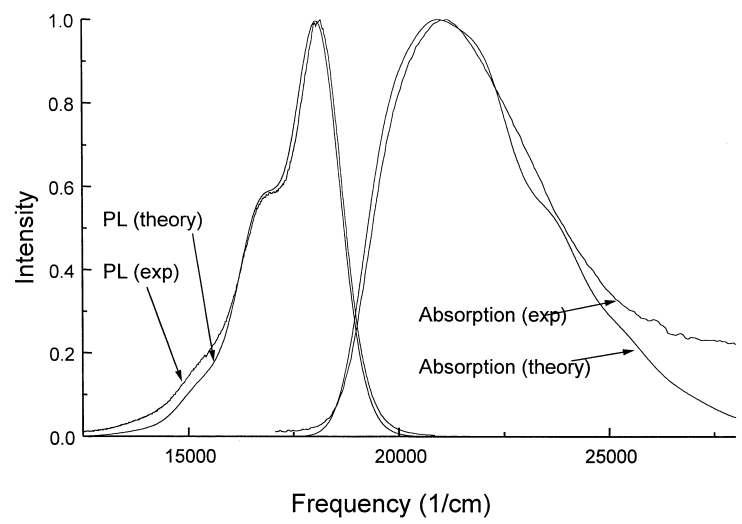

Fig. 1. Experimental (dashed lines) and calculated (solid lines) absorption and PL spectra of the DOO-PPV polymer in chloroform. Inset: Structure of DOO-PPV.

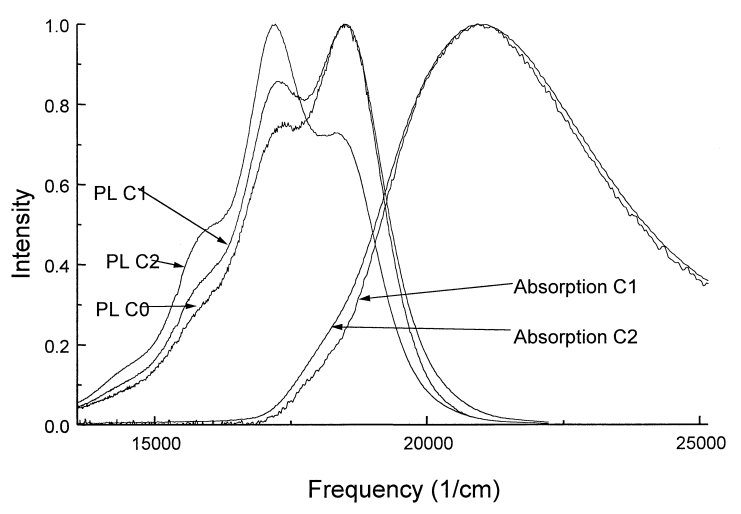

Fig. 2. Experimental absorption and photoluminescence spectra of DOO-PPV polymer in $\mathrm{m}$-THF with the monomer unit concentration being $c_{0}=10^{-6}, c_{1}=10^{-5}$ and $c_{2}=10^{-4} \mathrm{M}$. There is no absorption spectrum for $c_{0}$. The $c_{1}$ is normalized to compare with $c_{2}$.

room temperature. The solution was prepared by freshly dissolving the sample in chloroform, which is a good solvent, and diluted by $\mathrm{m}$-THF. The solution of m-THF was first heated to $60^{\circ} \mathrm{C}$ to increase the solubility, and cooled down to ambient temperature for experimental studies.

The absorption spectra were recorded on a Hitachi U-3200 spectrophotometer under ambient conditions. The fluorescence and excitation spectra were recorded by a SPEX Fluorolog-3 Spectrofluorometer. Time-resolved fluorescence was measured by a time-correlated single-photon-counting apparatus with a time resolution of about $80 \mathrm{ps}$. The excitation power used was less than $0.5 \mathrm{~mW}(\sim 7 \mathrm{pJ}$ per pulse) to avoid photo-damage of the samples.

Fig. 1 presents the absorption and PL spectra of DOO-PPV polymer in a 'good' solvent (chloroform). They resemble what we observed in MEH-PPV polymer solution with a slight change in character [23]. While DOO-PPV is in the 'poor' solvent $\mathrm{m}$ THF, the feature of the spectra changes. Fig. 2 illustrates the spectra in the m-THF solution for concentrations $c_{0}=10^{-6}, c_{1}=10^{-5}$, and $c_{2}=10^{-4}$ $\mathrm{M}$ monomer units. No absorption spectrum is taken for $c_{0}$ because the optical density is too low. According to Fig. 2, increasing the concentration of the solution leads to variations in the absorption spectra at the red edge. Moreover, the PL spectra undergoes greater variations in the spectral shape on the red side. More specifically, the magnitude of the peak at 

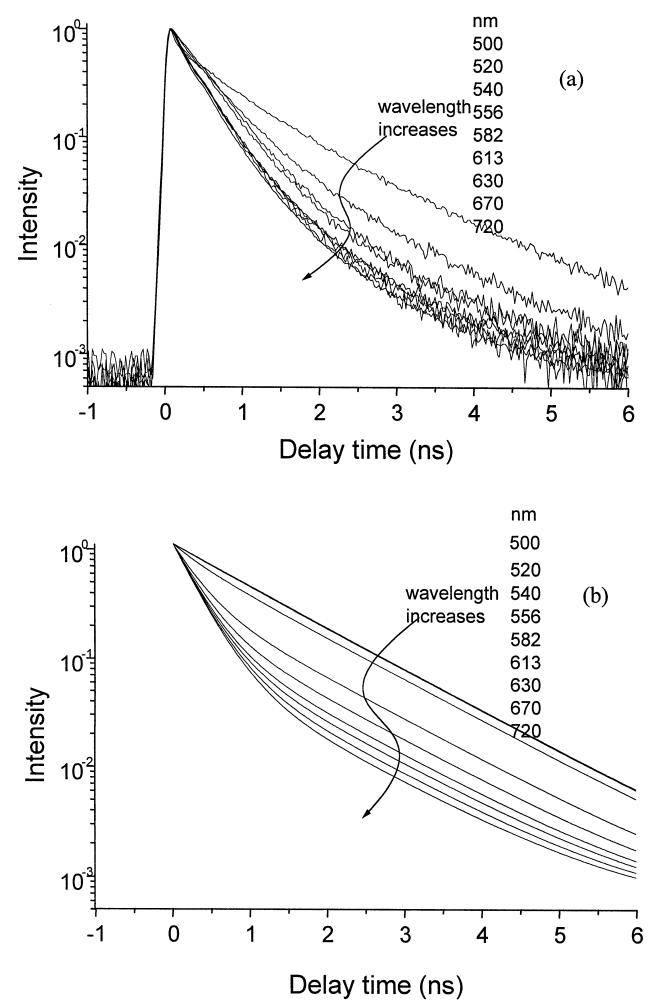

Fig. 3. Experimental (a) and calculated (b) time-resolved PL at different probe wavelengths for $c_{2}$.

about $17450 \mathrm{~cm}^{-1}$ largely increases with the concentration while that of the peak at $18750 \mathrm{~cm}^{-1}$ decreases with the concentration. For the solution of $c_{2}$, we also take time-resolved PL, and spectral dynamics is observed as shown in Fig. 3a. The decay rate increases with the wavelength. From all this spectral information, we infer that multi-emission species coexists in the poor solution.

\section{Theoretical analysis and results}

In order to unambiguously extract the properties of the interchain state, a rigorous understanding of 'intrachain' has to be made. Thus we will first analyze the spectra of DOO-PPV in chloroform as shown in Fig. 1. A recent study [23] models a conjugated polymer as a consecutive series of short oligomers. The distribution of oligomer chain lengths is described by a Gaussian function while the energy of excited states of each oligomer is simulated on the basis of exciton theory. The spectral profile of oligomer depends on its chain length and vibronic coupling constant. The same principle is used here to fit the experimental results as shown in Fig. 1 in which the chosen Gaussian distribution is peaked at 5 units of monomers with a width equal to 1.8 units.

With the intrachain information prepared, we may examine the interchain state from the variation of spectral features observed in high concentration solution in m-THF. The red edge broadening with concentration in absorption shown in Fig. 2 is related to the formation of aggregated states. The corresponding PL changes are consistent with this explanation. In the following, we assume that there exist only one kind of aggregated and intrachain state in poor solution. We will estimate the concentration ratio of the intrachain and aggregated species from the experimental results. Therefore the absorption spectrum is expressed as

$\alpha_{\text {total }}(\omega)=A_{m}\left|\vec{\mu}_{m}\right|^{2} \alpha_{m}(\omega)+A_{a}\left|\vec{\mu}_{a}\right|^{2} \alpha_{a}(\omega)$

where $A_{m}\left(A_{a}\right)$ and $\vec{\mu}_{m}\left(\vec{\mu}_{a}\right)$ are the concentration and effective transition moment of intrachain (aggregated) species, respectively. $\alpha_{m}(\omega)$ and $\alpha_{a}(\omega)$ are the corresponding normalized absorption coefficients (i.e. $\int \alpha_{m}(\omega) d \omega=1$ and $\int \alpha_{a}(\omega) d \omega=1$ ). In order to calculate the PL spectra and the time-resolved PL we construct a kinetic model

$$
\begin{aligned}
& \frac{\mathrm{d} \rho_{m}(t)}{\mathrm{d} t}=-k_{a m} \rho_{m}(t)-\Gamma_{m} \rho_{m}(t)+S_{m} \\
& \frac{\mathrm{d} \rho_{a}(t)}{\mathrm{d} t}=k_{a m} \rho_{m}(t)-\Gamma_{a} \rho_{a}(t)+S_{a}
\end{aligned}
$$

where $\rho_{m}(t)\left(\rho_{a}(t)\right)$ is the population of exciton on intrachain (aggregated) state, $\Gamma_{m}\left(\Gamma_{a}\right)$ denotes the corresponding intrinsic decay rate, and $k_{a m}$ indicates the energy transfer rate from intrachain to aggregated species. In Eqs. (2) and (3), the source terms $S_{m}=$ $A_{m}\left|\vec{\mu}_{m}\right|^{2} \quad \alpha_{m}\left(\omega_{\text {pump }}\right)$ and $S_{a}=A_{a}\left|\vec{\mu}_{a}\right|^{2} \quad \alpha_{a}\left(\omega_{\text {pump }}\right)$ are due to the excitation. Notably, $\omega_{\text {pump }}=25000$ $\mathrm{cm}^{-1}(400 \mathrm{~nm})$ is used herein. The time-resolved PL spectra can be expressed as

$$
I(\omega, t)=\sum_{s=a, m} \rho_{s}(t)\left|\vec{\mu}_{s}\right|^{2} I_{s}(\omega)
$$

where $I_{m}(\omega)$ and $I_{a}(\omega)$ are the normalized PL spectral profiles of intrachain and aggregated states, re- 
spectively. By using Eqs. (2) and (3), we can obtain the steady-state solution for $\rho_{s}(t)=\bar{\rho}_{s}$. The cw PL spectra can thus be calculated as

$I_{\text {total }}(\omega)=\sum_{s=a, m} \bar{\rho}_{s}\left|\vec{\mu}_{s}\right|^{2} I_{s}(\omega)$.

We assume that the absorption and PL spectra $\alpha_{m}(\omega)$ and $I_{m}(\omega)$ of the intrachain state are the same as the evaluated spectra in Fig. 1 except for a slight shift due to the change of solvent. However, a rigorous determination of $\alpha_{a}(\omega)$ and $I_{a}(\omega)$ is difficult; instead we use a simple approach. The model of randomly distributed oligomers has only been proved successful in the intrachain state, but we still assume that this model holds true in evaluating $\alpha_{a}(\omega)$ and $I_{a}(\omega)$. One minor modification is that the resonance frequency of each oligomer is red-shifted by 1300 $\mathrm{cm}^{-1}$; the other modification is that the vibronic coupling constant of each oligomer is increased slightly. The resulting profiles $\alpha_{a}(\omega)$ and $I_{a}(\omega)$ are illustrated in Fig. 4. Afterwards, merely intrachain and one aggregated states are sufficient to determine the total spectra $\alpha_{\text {total }}(\omega)$ and $I_{\text {total }}(\omega)$ for both concentrations $c_{1}$ and $c_{2}$. In other words, $A_{a}\left|\vec{\mu}_{a}\right|^{2} /$ $A_{m}\left|\vec{\mu}_{m}\right|^{2}$ and $\bar{\rho}_{a}\left|\vec{\mu}_{a}\right|^{2} / \bar{\rho}_{m}\left|\vec{\mu}_{m}\right|^{2}$ are the only parameters. By using Eq. (1) to fit the absorption, as shown in Fig. 5a,b, the values of one of the two parameters are determined as $A_{a}\left|\vec{\mu}_{a}\right|^{2} / A_{m}\left|\vec{\mu}_{m}\right|^{2}=0.4(0.8)$ for $c_{1}\left(c_{2}\right)=10^{-5}\left(10^{-4}\right) \mathrm{M}$.

Prior to determining the parameter $\bar{\rho}_{a}\left|\vec{\mu}_{a}\right|^{2} /$ $\bar{\rho}_{m}\left|\vec{\mu}_{m}\right|^{2}$ to fit cw PL, we analyze the time-resolved

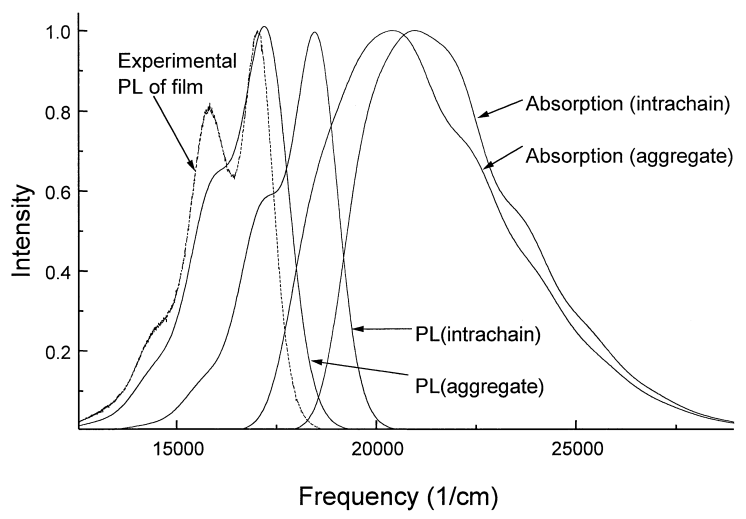

Fig. 4. Calculated absorption and PL spectra for intrachain (same as Fig. 1) and aggregated states in m-THF. The dashed line is the experimental PL for DOO-PPV film.
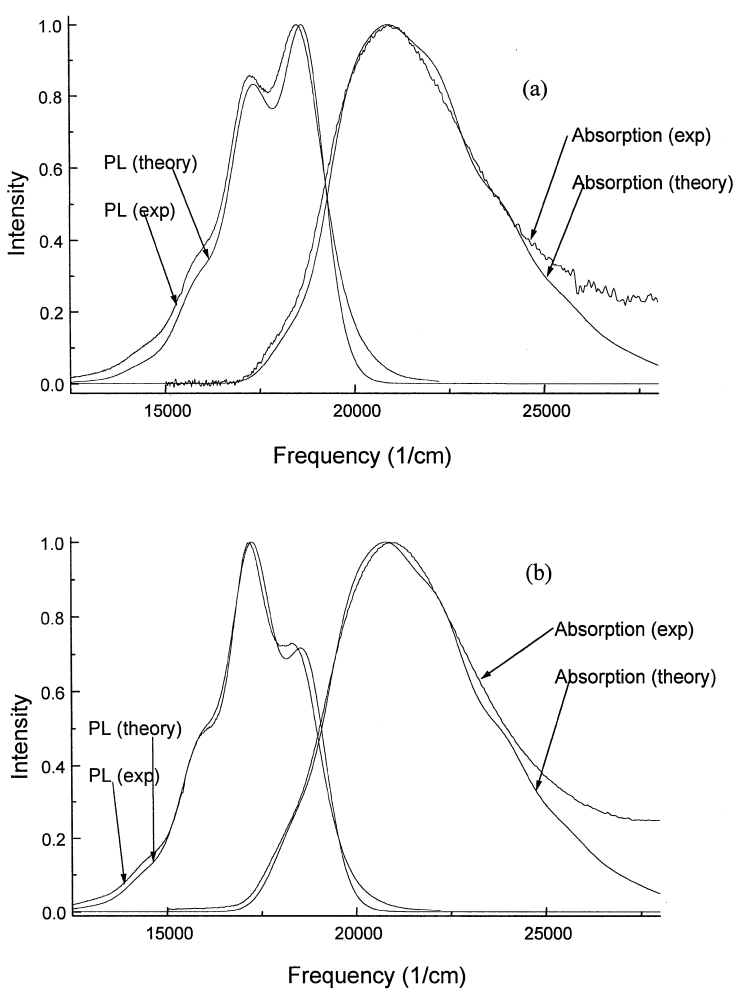

Fig. 5. Experimental and calculated absorption and PL spectra of DOO-PPV in m-THF of $c_{1}$ (a) and $c_{2}$ (b).

PL of concentration $c_{2}$ (shown in Fig. 3a). Fig. 3b shows the evaluated time-resolved PL of several wavelengths, using Eqs. (2)-(4). We have chosen $\left|\vec{\mu}_{a}\right|^{2} /\left|\vec{\mu}_{m}\right|^{2}=3.0,1 / \Gamma_{m}=1.2 \mathrm{~ns}, 1 / \Gamma_{a}=0.3 \mathrm{~ns}$ and $k_{a m}=0.2 \mathrm{~ns}^{-1}$ in the calculation. By using these parameters, we have obtained $\bar{\rho}_{a} / \bar{\rho}_{m}=1.2$ from the steady state solution of Eqs. (2) and (3), which allows us to evaluate the cw PL spectra by Eq. (5). The result is shown in Fig. 5b. As for $c_{1}$, we use the same parameter values except $k_{a m} \sim 0.02 \mathrm{~ns}^{-1}$ which leads to $\bar{\rho}_{a} / \bar{\rho}_{m}=0.72$ and the evaluated $\mathrm{cw}$ PL spectrum is shown in Fig. 5a. The small value of $k_{a m}$ indicates that the solution of $c_{1}$ is not dense enough to have the energy transfer take place. If we assume that the decay of the intrachain state is primarily from a radiative process, the choice of $\left|\vec{\mu}_{a}\right|^{2} /\left|\vec{\mu}_{m}\right|^{2}=3.0$ will give a radiative lifetime of aggregated state $1 / \Gamma_{a}=0.4$ ns quite close to the estimation $1 / \Gamma_{a}=0.3 \mathrm{~ns}$. The small deviation could be correlated with the additional radiationless decay 
such as formation of an interchain polaron pair or excimer. It follows that the aggregated state has a quantum efficiency 0.75 relative to the intrachain state. Recent experimental work by Hsu et al. [19] shows that the quantum yield of aggregate in toluene is about half of the single-chain polymer that qualitatively agrees with our estimation. Moreover, we find the emission spectra of thin film (as shown in Fig. 4) and aggregated state start at frequencies quite close to each other. Thus it can be deduced that the main part of the emission in thin film is contributed by the aggregated state that we have observed in poor solution. This viewpoint is also consistent with the experimental result obtained by Hsu et al. [19]. Since thin film usually has a quantum efficiency much less than the aggregated state [6,9], we have deduced that it should contain some states that are less emissive and quench the PL effectively.

\section{Discussion}

This work has rationalized the experimental result by assuming that an aggregated state exists in poor solutions. The spectral change induced by a high concentration is attributed mainly to the relative weighting between the intrachain and aggregated state. The decay time of the aggregated state is about $0.3 \mathrm{~ns}$ which is related to its much larger oscillator strength, i.e. $\left|\vec{\mu}_{a}\right|^{2} /\left|\vec{\mu}_{m}\right|^{2}=3.0$. However, one should note the point given herein. The simplest kind of aggregated state is a dimer which has maximum oscillator strength $\left|\vec{\mu}_{a}\right|^{2}=2\left|\vec{\mu}_{m}\right|^{2}$. There are two possible reasons that account for the larger oscillator for aggregated state. First, during the formation of a dimer between different chains, the torsional motion of each chain is also reduced. Consequently, the effective conjugation length of each chain increases, the transition moment also increases correspondingly, and the total transition moment of the dimer is likely to be greater than $2\left|\vec{\mu}_{m}\right|^{2}$. Second, the emissive state could be a trimer or larger aggregation that a larger transition moment is naturally anticipated. Although our analysis can fit the experimental result using two species model, the other species might still exist. Notably our empirical profile for the aggregated state has a red shoulder at $\sim 16000 \mathrm{~cm}^{-1}$ which has a magnitude slightly higher than antici- pated (see Fig. 4) because aggregated states usually have weaker vibronic peaks [23]. Thus this shoulder could correspond to some other species with lower energy and low quantum efficiency. In the limiting case of thin film, the shoulder is more pronounced (also shown in Fig. 4), indicating that excimer states exist in the lower-energy region. Jakubiak et al. [24] ascribe these lower excimer states to the indirect excitons that largely reduce the total quantum efficiency of PL. Nevertheless, this contribution is less in the poor solution and should not invalidate our analysis.

In summary, the concentration-dependent spectra confirm the existence of an aggregated state. This state has a substantially lower electronic energy and significant quantum efficiency. By resembling the PL spectra, the aggregated state is identified to be the main emission species in thin film, while we also deduce that some non-emissive states exist in thin film, leading to the extremely low quantum efficiency.

\section{Acknowledgements}

This work is supported in part by NSC in Taiwan and in part by Academia Sinica, Taiwan.

\section{References}

[1] P.L. Bum, et al. Nature 356 (1992) 47.

[2] G. Gustafsson, et al. Nature 357 (1992) 477.

[3] N.C. Greenham, S.C. Moratti, D.D.C. Bradley, R.H. Friend, A.B. Holmes, Nature 365 (1993) 628.

[4] N.C. Greenham, R.H. Friend, in: H. Ehrenreich, F.A. Spaepen (Eds.), Solid State Physics 49, vol. 2, Academic, San Diego, CA, 1995.

[5] M. Yan, L. Rothberg, B.R. Hsieh, R.R. Alfano, Phys. Rev B. 49 (1994) 9419.

[6] M. Yan, L. Rothberg, F. Papadimitrakopolous, M.E. Galvin, T.M. Miller, Phys. Rev. Lett. 72 (1994) 1104.

[7] M. Yan, L. Rothberg, E.W. Kwock, T.M. Miller, Phys. Rev. Lett. 75 (1995) 1992.

[8] N.T. Harrison, G.R. Hayes, R.T. Phillips, R.H. Friend Phys, Rev. Lett. 77 (1996) 1881.

[9] N.C. Greenham, I.D.W. Samuel, G.R. Hayes, R.T. Phillips, Y.A.R.R. Kessener, S.C. Moratti, A.B. Holmes, R.H. Friend, Chem. Phys. Lett. 241 (1995) 89.

[10] V. Klimov, D. Mcbranch, N.N. Barashkov, J.P. Ferraris, Chem. Phys. Lett. 277 (1997) 109. 
[11] L. Rothberg, F. Papadimitrakopoulos, M. Galvin, Synth. Met. 80 (1996) 41.

[12] E.M. Conwell, J. Perlstein, S. Shaik, Phys. Rev. B 54 (1996) R2308.

[13] U. Lemmer, S. Heun, R.F. Mahrt, U. Scherf, M. Hopmeier, U. Siegner, E.O. Göbel, K. Mullen, H. Bässler, Chem. Phys. Lett. 240 (1995) 373.

[14] I.D.W. Samuel, G. Rumbles, C.J. Collison, Phys. Rev. B 52 (1995) 11573.

[15] G.R. Hayes, I.D.W. Samuel, R.T. Phillips, Phys. Rev. B. 54 (1996) R8301.

[16] I.D.W. Samuel, G. Rumbles, C.J. Collison, S.C. Moratti, A.B. Holmes, Chem. Phys. 227 (1998) 75.

[17] J.F. Grüner, R.H. Friend, U. Scherf, J. Huber, A.B. Holmes, Adv. Matter 6 (1994) 748.
[18] S.A. Jenekhe, J.A. Osaheni, Science 265 (1994) 765.

[19] J.H. Hsu, W.S. Fann, P.S. Tsao, K.R. Chuang, S.A. Chen, J. Phys. Chem. A 103 (1999) 2375.

[20] C.L. Gettinger, A.J. Heeger, J.M. Drake, D.J. Pine, J. Chem. Phys. 101 (1994) 1673.

[21] G.J. Sarneck, P.L. Burn, A. Kraft, R.H. Friend, A.B. Holmes, Syn. Met. 55/57 (1993).

[22] S.H. Askari, S.D. Rughoophth, F. Wudl, Syn. Met. 29 (1989).

[23] R. Chang, J.H. Hsu, W.S. Fann, K.K. Liang, C.H. Chang, M. Hayashi, J. Yu, S.H. Lin, E.C. Chang, K.R. Chuang, S.A. Chen, Chem. Phys. Lett. 317 (2000) 142.

[24] R. Jakubiak, C.J. Collison, W.C. Wan, L. Rothberg, B.R. Hsieh, J. Phys. Chem. A 103 (1999) 2394. 